\title{
WIRELESS GRIDS: Assessing a New Technology from a User Perspective
}

\author{
Lee W. McKnight \\ Raed M. Sharif \\ Syracuse University \\ Syracuse, NY U.S.A. \\ Lidwien van de Wijngaert \\ Utrecht University \\ Utrecht, The Netherlands
}

\begin{abstract}
The objective of this paper is to assess the value of wireless grids from the perspective of users. In a ubiquitous information environment, wireless grids allow the ad hoc sharing of resources (e.g., microphones, screens, processing power) of edge devices (e.g., mobile phone, laptop, $P D A$ ). Wireless grids are one of the emerging wireless communication concepts that have been developed in university and industry research laboratories. So far, literature about wireless grids has tackled some of the technical and policy issues about the technology. This paper provides the first empirical study about wireless grid technology from the user perspective.

Using Rogers' diffusion of innovations model, this paper focuses on the future diffusion of this technology. Using the results of two focus group meetings, we suggest that the introduction of the technology and its future diffusion will be a complex process. The future acceptance and use of this technology requires not only social and mental changes to move from one stage to another in the diffusion process, but also changes in the coordination and pricing mechanisms, and even changes in the technology itself.
\end{abstract}

\section{INTRODUCTION}

"Wireless communications is a rapidly evolving and promising sector of the communications arena, and even in a challenging time for the telecommunications industry, 
represents a significant development opportunity for companies and organizations in creating a global market" (McKnight et al. 2002, p. 11). The products and services of this sector, mainly wireless devices, are increasing in numbers, and their power is growing (Gaynor et al. 2003). People are increasingly using wireless devices in their professional and personal lives. Different research and development efforts are taking place in industry and universities to further develop these devices and find new potentials for their use. One of the new emerging concepts of wireless communications is wireless grids. As the concept of wireless grids is new, we will first provide a description of what the technology encompasses. After that, we will present the goals of our research and the results in the rest of this paper.

\subsection{Wireless Grids}

According to McKnight et al. (2004), "Wireless Grids is a new type of resource sharing network that connects sensors, mobile phones, and other edge devices with each other and with the wired grid. Ad hoc distributed resource sharing allows these devices to offer new resources and locations of use for grid computing" (p. 24).

The ultimate vision of the grid is that of an adaptive network offering secure, inexpensive, and coordinated real-time access to dynamic, heterogeneous resources, potentially traversing geographic, political and cultural boundaries but still able to maintain the desirable characteristics of a simple distributed system, such as stability, transparency, scalability and flexibility (McKnight et al. 2002, p. 2).

To give more insight about the nature of this technology and its use, we present a number of wireless grids applications that were developed by the wireless grids research team at Syracuse University. These application can be used both in an organizational as well as an end user context.

\subsubsection{Distributed Audio Recording}

Most small devices have the ability to record simple audio. Cell phones, PDAs, and most laptops have a simple microphone built in. The problem with these simple audio recording devices is that they produce simple audio. They don't produce stereo sound, and they might not pick up subtle sounds in the background, like a person talking on the other side of a room.

What distributed audio recording application does is build a small wireless network of these devices and then share the recorded audio between them. By combining the audio from these different devices, it is possible to make a stereo recording. Another possibility is to combine several streams in a lecture or office meeting and get a good recording of questions without having to pass around a microphone or have people aim their conversation toward a tape recorder.

Figure 1 provides a graphical outline of the service architecture for the distributed audio recording and other wireless grid applications. 


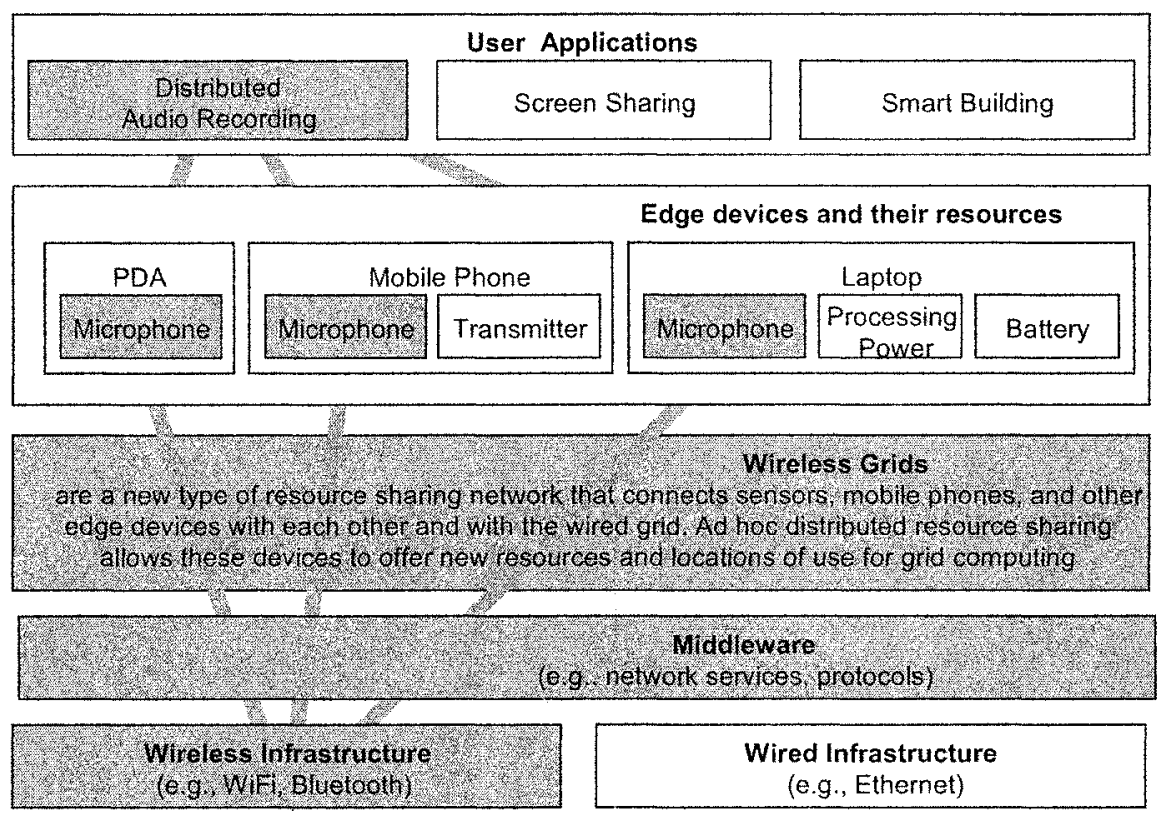

Figure 1. Wireless Grid Architecture

There are two resources being shared with the distributed audio recording application: a recorder resource and a mixer resource. The recorder is simply a resource that will capture a live audio stream using a microphone of some kind. The mixer resource can take advantage of multiple recorder resources and mix them together to create a unified audio stream.

\subsubsection{Wireless Ad Hoc Screen Sharing}

Another example of a wireless grid application can be found at a typical business meeting where more than one person will be presenting something, using slides. Often, each person has their own laptop which contains the presentation, and people take turns connecting their machines to a projector. The projector tends to become a bottleneck because each person who wants to present using the projector needs to (1) hook up their laptop to the projector and (2) find and change the settings needed to use the projector. While this may not seem like a big ordeal, it gets to be more of a problem when there are more people making presentations (for example, at academic conferences). The wireless ad hoc screen sharing application gets rid of having to make a physical connection to a projector by essentially sharing the projector with one or more different laptops over a wireless network.

In order to share a projector, two different pieces of software are needed. The first software, the display manager, resides on a single machine that is hooked up to the 
projector. This software is in charge of deciding who is the current "owner" of the projector. The second piece of software, the display client, runs on the laptop of anyone who wants to use the projector. The two pieces of software talk to each other over a wireless network and share the graphics shown on a given screen (McKnight et al. 2004).

\subsubsection{Smart Buildings and Location Aware PDAs}

A third example of a wireless grid application is that of a PDA connecting to the wireless network inside a building. Not only does it connect to the wireless network, but also it becomes a location-aware device within the building. It shows a map of the building, with an indicator of where you are on that map. Using the building intranet site on the PDA, it is possible to retrieve directions and announce arrival, for example, by using voice over IP (VoIP) technology to turn your PDA into a local communicator.

\subsection{Research Goal}

During the past decade, the concept of wireless grid technology has emerged in different industrial and academic labs (e.g., Intel Research at Berkeley and the TeleCom City project of Syracuse University, MIT, Tufts, and Boston University). Most of this research has focused on the technical challenges and developments of wireless grids. Although theoretical work has been done in the areas of economic and future market challenges of wireless grids; no empirical studies were directed to the potential individual users themselves to hear about their perceptions, beliefs, attitudes, and concerns about this technology. Gaynor et al. (2003) and McKnight et al. (2002) suggest that further research is needed in the area of the economic and market challenges of wireless grid technology.

This empirical study is the first of its kind in the area of wireless grid technology where empirical evidence about the future use of this technology by individual users was collected. Using the basic elements of Rogers' (1995) model for the diffusion of innovations, our aim is to assess the value of wireless grids outside the laboratory from a user perspective and its potential diffusion model.

We will proceed by describing our theoretical framework, which strongly draws on the work of Rogers. In section 3, we will describe how two focus group meetings were set up and analyzed. Section 4 describes the results from these focus groups and, in section 5, we will discuss the conclusion and limitations of the research and opportunities for further research.

\section{DIFFUSION OF INNOVATIONS}

Several theories help to explain the adoption and use of new technologies: diffusion of innovations (Rogers 1995), domestication (Silverstone and Haddon 1996), sense 
making (Dervin 1986), and (unified) technology adoption model (Davis 1989; Venkatesh et al. 2003) are just a few examples of the many possible theoretical frameworks. Although we are aware of the fact that each of these theories, models, and frameworks can provide important insights with regard to the adoption and use of wireless grids, we chose to start this paper, the first that investigates wireless grids from a user rather than a technical perspective, with Rogers' classic work on the diffusion of innovations.

Rogers' work on the diffusion of innovations provides us with a starting point to understand how wireless grids might diffuse through time and space. Rogers defines the diffusion of innovations as "is the process by which an innovation is communicated through certain channels among the members of a social system" (p. 5). Looking from the perspective of individual-level adoption decisions, Rogers identifies five characteristics of the innovation that can either facilitate or impede the adoption of an innovation.

- Relative advantage is the degree to which the innovation is perceived as being better than the idea it supersedes. The degree of relative advantage is, for example, expressed in economic profitability or social status.

- Compatibility is the degree to which an innovation is perceived as consistent with the existing (socio-cultural) values, past experiences with previously introduced ideas, and the need for innovations by potential adopters.

- Complexity is the degree to which an innovation is difficult to understand and use.

- Triability is the degree to which an innovation can be experimented with on a limited basis.

- Observability is the degree to which the benefits of the proposed innovation are visible.

Besides these characteristics of the innovation, Rogers mentions numerous other factors that explain the rapid diffusion of an innovation. These factors not only relate to the individual's decision to adopt or reject an innovation, but place it in a broader context. Rogers, for example, mentions the type of innovation decisions and the way in which communication channels and change agents function.

In this paper, we will also address another important factor in the diffusion process: diffusion networks. The S-shaped curve is used to describe the process of more and more people joining in the innovation. In the beginning of the process, diffusion is slow and the number of new users is small. Oliver et al. (1985) argue that people who adopt an innovation in this phase choose to make a big contribution to the collective action while the majority do little or nothing. Once a "critical mass" is reached, Markus (1987) states that enough individuals have adopted an innovation so that the innovation's further rate of adoption becomes self-sustaining. In the last phase of the S-shaped adoption curve, the number of new users again diminishes. Here the market is reaching saturation: the moment in which the maximum number of adopters is reached. This does not necessarily need to be 100 percent of the population, but rather 100 percent of the potential adopters. In addition, the traditional S-shaped curve changes when the innovation is an interactive service (like telephone, fax, e-mail, and wireless grids). Economides (1991) states that network externalities occur when "the buyer of the last unit of a good has a higher benefit than the buyer of the first unit because the sale of the 
earlier units has created some benefit in a related dimension." In other words, network externalities are a quality of certain goods and services such that they become more valuable to a user as the number of users increases. This makes reaching a critical mass even more difficult than for products that do not have network externalities.

\section{RESEARCH METHOD}

Our research is aimed at obtaining insight into the starting position of the technology. As mentioned above, the main goal of this study is to predict the future diffusion model of wireless grid technology through listening to the thoughts and concerns of potential individual users about this new technology. Therefore, we have performed two focus group meetings. Volunteers for the focus groups were recruited through email and personal invitations. The invitations were sent to three categories: faculty members, staff members, and graduate students (Ph.D. and Master's levels). Twenty volunteers were divided into two separate focus group meetings. Because it is a new technology and given the lack of awareness about this technology and its potential use, it was decided to start the meetings with a 20 minute introductory presentation about the technology with another 20 minutes to answer the participants' questions. During the 1-hour discussion, the participants were asked the same questions, which focused on the potential individual user's perceptions, attitudes, concerns, and future intentions regarding wireless grid technology.

The video recordings of the focus groups were transcribed and entered into the content analysis software Kwalitan (Peters 2004). This software allows systematic ordering of open, qualitative, unstructured data. The data from focus group meetings were analyzed using content analysis and a coding scheme developed by the researchers. The coding scheme was based on the theoretical framework presented earlier in this paper.

\section{RESULTS}

In this section, we will provide an overview of the discussion that took place during the focus group meetings. We will examine how wireless grids as an innovation hold up to the factors that were enumerated by Rogers.

\subsection{Relative Advantage}

From a very theoretical and abstract point of view, the participants' could see many values and benefits in using this technology. Their first and immediate reaction and reflections on the technology consisted of a strong agreement that using this technology is valuable especially in terms of time and effort saving and in providing them with new resources and capabilities. The participants went even further and provided different scenarios where sharing resources through the wireless grid technology might help in making their lives easier and more efficient. 
It's very promising technology. The resources are extensive; there are lots of things to share and the case of cell phone is one of them. Many times you have the cell phone but don't have the signals and it is a common problem these days. If I am next to someone with a signal, which is a common situation too, given that we have different cell phones companies with different coverage capacities, and my cell phone will immediately pick the signal from this person, this is all what I need, at the end of the day I want to use the cell phone to have my work done.

Yes, I think it is absolutely useful. For example, I am still carrying my laptop wherever I go to do my work, which is still very heavy. If we reduce the size to the PDA and I can do everything on the PDA including my homework, for example, through sharing others' resources, this will definitely help me and make my life easier.

Wow, this seems to be very cool technology and will allow me to be more mobile with my smart devices.

However, from a practical point of view, especially after discussing the use of this technology in real-life scenarios and after being asked if they would share their resources in real-life cases under different circumstances, the participants started to realize and identify some concerns might be associated with the use of this technology. The focus of these concerns was mainly on security, privacy, and trust issues. In the context of wireless grid technology, the participants' security concerns were mostly related to loss of information, information and/or device damage, and other security consequences.

For me, the security will be a very big concern; it is just related to the human tendency. For example, if I have this small device and people are going to request the processing power of my device, I am going to worry about viruses and other security and privacy issues, so to reduce this complexity, I would probably say no.

If people ask me to share some of my resources, I might say no. Yes, no because this is my device and I don't want to run into technical problems later because I let people use it.

I definitely think that security would be an issue. I guess I should be able to choose whom I can give or share information with. That would make me feel so much safer.

\subsection{Compatibility}

Using IT is almost synonymous with fast change. In general people are used to the fact that new technologies build on existing ones (e.g., a mobile phone with an Internet 
connection, a laptop with a wireless connection, a PDA that can send e-mail). A mobile phone that can use the signal of another phone or a laptop that can share a screen with another laptop seems like a logical next step. In that sense, the use of wireless grids seems like an innovation that is very compatible with other existing technologies.

However, the trust and privacy issues that were mentioned by the participants strongly relate to the concept of compatibility. Sharing a personal item like a laptop or mobile phone is something that would require at least a mental and social change of how we perceive devices and their resources as our own.

How people will be able to protect their personal and private information and what will make sure that this personal information will not be accessed and/or stolen were among the participants' questions and concerns when using wireless grid technology and sharing their resources with others. Some of the reactions to this issue were extreme and tended toward total rejection, which was also reflected by one participant's body language and signs of fear of losing something.

Of course I will never let anybody share my resources and to give them the chance to access my private and personal stuff. Oh my God! It's my privacy.

Others, however, expressed moderate but conditional reactions and responses and they seemed to be willing to compromise some of their security and privacy in case the technology would provide some solutions.

Yes, I fully agree that sharing resources with others will be very valuable, but I think the privacy and ownership issues will be of very special concern not only for me, but for most of the people I guess.

I am OK in this community and this environment of sharing resources, but I would need somewhere in my computing world where I could block some of my stuff away so not everything is accessible in every way. I think that there has to be identified levels of accessibility.

Participants put a high level of trust as an important condition to share resources with others; they also suggested that different levels of trust could be found in this kind of relationships. The participants identified two levels of trust. Trust at a personal level (e.g., family and friends) and trust at a professional level (e.g., work colleagues).

\subsection{Complexity}

Of special concern for the participants about sharing resources with others and the consequences of this process is the pricing and coordination mechanism that will coordinate, regulate, and manage the way people use and deal with this technology.

The question is not whether it is valuable or not, the question is what is the best business model that can make it work? 
I don't want to personally negotiate with people the sharing of resources, I just want to know what is available and to have it, there must be a system that manages this process, If I need to get into negotiations, then it is not worth my time.

Another thing that would also be of concern is the price factor. How much I will charge? For what service? And how to get the money? These are questions need to be answered.

So, as long as the sharing is limited within a small and known community or work group context, complexity will be relatively low as the people that want to share are trusted friends or colleagues. But when the use of wireless grids grows and enters the public space, complexity increases considerably. Once outside a small community of friends or colleagues, it will be quite complex to find a model in which people are willing to share their resources and still understand the system. People may be willing to share, if there is something in it for them as well. A business model in which people can exchange credits or real money may be too much to ask, at least at the early stages of the diffusion process. So people will need to find a balance between security (allowing strangers to use their device), the degree of disruptiveness and the risk of losing valuable assets, and the possible benefits of sharing.

\subsection{Triability}

When asked about possible solutions for the problems and concerns they raised, and as an indicator of the willingness of people to balance the potential cost (financial and nonfinancial) and benefits (financial and nonfinancial) of the technology, the participants suggested starting to use wireless grid technology in communities. These communities with, to a large extent, known and trusted members (e.g., project teams, faculty members, doctors) can serve as a role model or a demonstration site of the technology for other future individual users. Within this community, where sharing resources will be free of charge, the participants believe the security and trust issues will not be a big concern.

I would want to be sure that I am sharing with only the people that I know and trust, for example my coworkers, and then once I am comfortable with it and comfortable with what is out there and what is happening, I probably would go to the next level and I would share with the public. There would be A-level users, and B-level users, etc.

After some period of time, where they will have the opportunity to test this technology with known and trusted people, the participants said that they can move to another level outside their community where other coordination, pricing, and payment mechanisms need to be identified. 


\subsection{Observability}

In order to see what observability means for wireless grids, we need to look at the technology from a broader perspective. Over the past few years, we have seen how mobile phones (and other small devices like PDAs and notebooks) have turned into fashion items (Fortunati 2001). New technologies are not only purchased for their functionality, but also as a social statement. Although many people will not actually use all these features, people like having the opportunity to use them, if only to show off to other people. Innovators and early adopters are especially sensitive to this opportunity (Rogers 1995). Wireless grids could be an addition to the list of capabilities of laptops, mobile phones, and PDAs. When adopting (and not actually using) the technology is a sufficient reason for purchasing it, one could argue that observability of the technology is very high. Peer-to-peer communication with regard to the advantages of wireless grids could then become an important factor in the fast diffusion of the technology. Finally, once people have the technology installed in a device, it is easy to play around and experiment with it in different situations, increasing the level of observability.

\subsection{Network Effects}

Finally, we want to focus on network externalities and the diffusion effect as an important factor in the fast diffusion of wireless grids. The use of wireless grid technology requires some other devices that also have this technology installed. This situation is unlike the use of a mobile phone which can be used to contact another mobile phone as well as a fixed phone. Because the installed base of fixed phones is very high, purchase of a mobile phone has immediate value. Early adopters of wireless grids are confronted with a lack of other users with whom to share their devices. This interdependence on the system level has a negative effect of the diffusion of wireless grids. The lack of a critical mass of users is a threat to the diffusion of wireless grids. This stresses the importance of the wireless grid as a social statement (no network externalities) and the use of Wireless Grids within small communities (critical mass is easily reached). That also reaffirms the importance of having good marketing strategies for the wireless grids, especially free trials and subsidized communities.

\section{CONCLUSION AND DISCUSSION}

\subsection{Limitations and Further Research}

This paper is based on the results of two focus groups of highly educated people who were familiar with the field of information technology. Although the level of subjectivity of the qualitative and interpretive approach we used is relatively high, and although it might be difficult to generalize the findings, this was one of the few relevant approaches, especially in the case of the first paper on this topic, to collect some insight into the future of this technology from the perspective of the user. 
We intend to follow further development of wireless grids. Currently, we are conducting a policy capturing study. The goal of this study is to obtain insight into the conditions under which people are willing to share their resources and to be confronted with some of their security, privacy, and trust concerns. By presenting respondents with different real-life scenarios, we can see in which cases people are or are not willing to share. Factors that are being studied, for example, are the degree of trust in the situation, the degree to which the request is related to an emergency or a regular situation, who benefits from sharing (only the person who wants to share or both), and the gender of the asker. Besides these situational characteristics, we also take user characteristics (such as gender, IT experience, and altruism) into account.

Furthermore, we will study a group of high school students who will be experimenting with wireless grids during a summer course in 2005. These students will be equipped with PDAs that are enabled for wireless grids. By closely watching how the process of mutual shaping develops, we will be able to draw conclusions not only on the diffusion of wireless grids, but also on the diffusion of future related new technologies.

\subsection{Conclusion}

Rogers' concept of relative advantage was clearly described by participants in the focus groups in terms of benefits of the technology. Other issues, such as trust, privacy, and security relate to Rogers' concepts of compatibility and triability. The respondents search for a viable business model also relates to Rogers' concept of complexity. Observability and network externalities, although important theoretical measures in the diffusion of wireless grids, were not explicitly mentioned by people in the focus groups.

From the findings we should conclude that the introduction of wireless grids is not a simple process. Drawing on theories that stress the introduction of new technology as a dynamic process, the authors suggest that people need to learn how to share their resources in a process of mutual adaptation (Williams and Edge 1996). Therefore, we argue that the introduction of wireless grids should follow a step-wise process. In this process, critical mass is not reached once, but several times, in different situations, and with different technologies (Bouwman et al. 1992). Figure 2 illustrates how we see this process.

We can also conclude that the technology has three immediate potential groups of users. The first group is the group of people who are already up to date with the latest wireless communication technologies (e.g., Wi-Fi and Bluetooth). Such a group of people might include, but not be limited to, engineers, managers, faculty members, students, and IT professionals. We believe that this group will be one of the easiest groups to target. The second group is teenagers, who are inclined to obtain new technologies in order to underline their social status. Based on the past examples of cell phones, digital cameras, and PDAs, we expect this group to behave similarly with regard to wireless grid technology and to show high interest in adding this technology to their other fashion technologies. A third opportunity is to introduce the technology within specific communities (e.g., hospitals, police, fire fighters). 


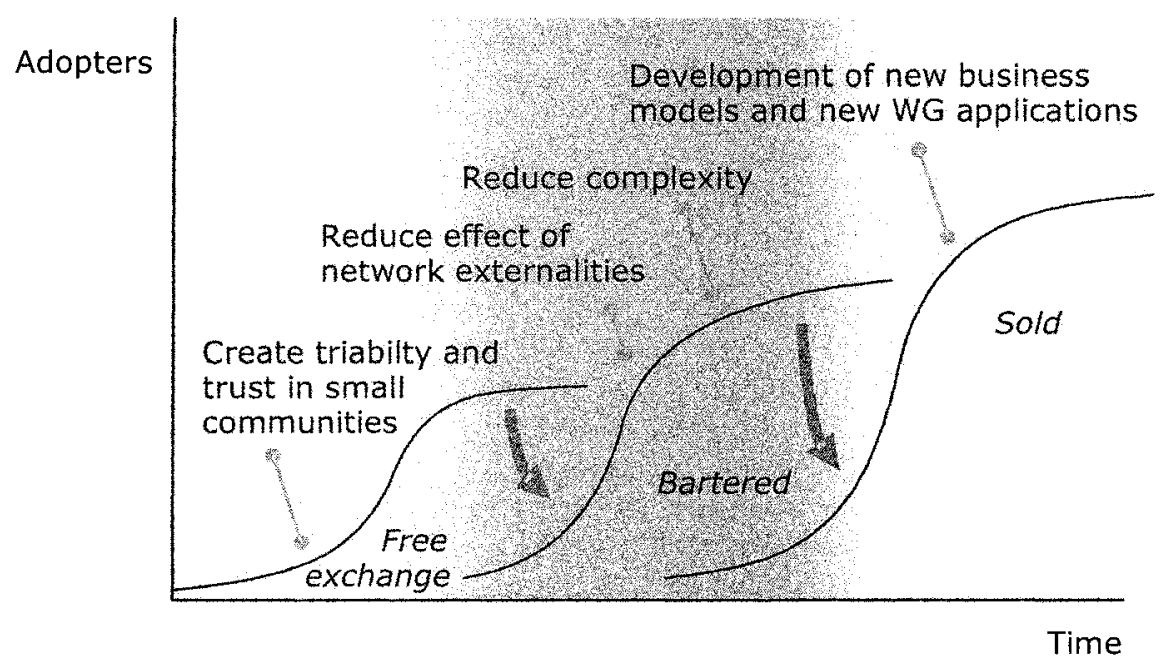

Figure 2. Mutual Shaping of Technology and Diffusion

On the diffusion road of wireless grids, there are many pitfalls that might prevent wireless grids from becoming successful. Lack of trust and network externalities are factors that need to be addressed with care. In this process, the technology will change and adapt itself to the new situation.

\section{REFERENCES}

Bouwman, H., Christoffersen, M., and Ohlin, T. "Introduction. Videotex: Is There a Life After Death?," in Relaunching Videotex, H. Bouwman and M. Christoffersen (Eds.), Dordrecht: Kluwer Academic Publishers, 1992, pp. 7-15.

Davis, F. D. "Perceived Usefulness, Perceived Ease of Use, and User Acceptance of Information Technology," MIS Quarterly (13:3), 1989, pp. 319-341.

Dervin, B. , and Nilan, M. "Information Needs and Uses," Annual Review of Information Science and Technology (21), 1986, pp. 3-33.

Economides, N. "Compatibility and the Creation of Shared Networks," Chapter 3 in Electronic Services Networks: A Business and Public Policy Challenge, M. E. Guerin-Calvert and S. S. Wildman (Eds.), New York: Praeger, 1991.

Fortunati, L. "The Mobile Phone: An Identity on the Move," Personal and Ubiquitous Computing (5:2), 2001, pp. 85-98.

Gaynor, M., McKnight, L., Hwang, J., and Freedman, J. "Wireless Grid Networks and Virtual Markets," in Proceedings of the International Conference on Computer, Communication and Control Technologies (CCCT '03)/ $9^{\text {th }}$ International Conference on Information Systems Analysis and Synthesis (ISAS '03), Orlando, FL, July 31-August 2, 2003 (CD-ROM).

Markus, M. L. "Toward a Critical Mass Theory of Interactive Media: Universal Access, Interdependence, and Diffusion," Communication Research (14:5), 1987, pp. $491-511$. 
McKnight L, Anius, D, and Uzuner, O. "Virtual Markets in Wireless Grids: Peering Policy Obstacles," paper presented at the TPRC $30^{\text {th }}$ Annual Research Conference on Communication, Information and Internet Policy, Alexandria, VA, September 2002 (available online at http:/tprc.org/papers/2002/88/virtualmarkets.pdf).

McKnight, L. W., Howison, J., and Bradner, S. "Wireless Grids: Distributed Resource Sharing by Mobile, Nomadic, and Fixed Devices," IEEE Internet Computing (8:4), July-August 2004, pp. 24-31 (available online at http://csdl.computer.org/comp/mags/ic/2004/04/ w4024.pdf).

Peters, V. Kwalitan: A Supportive Program for Qualitative Analyses, Version 5.0, Nijmegen, The Netherlands: Katholieke Universiteit Nijmegen, 2004.

Oliver, P., Marwell, G., and Teixera, R. "A Theory of the Critical Mass, I: Interdependence, Group Heterogeneity, and the Production of Collective Action," American Journal of Sociology (91), 1985, pp. 522-556.

Rogers, E. M. Diffusion of Innovations, New York: Free Press, 1995.

Silverstone, R., and Haddon, L. "Design and the Domestication of Information and Communication Technologies: Technical Change and Everyday Life," in Communication by Design: The Politics of Information and Communication Technologies, R. Mansell and R. Silverstone (Eds.), Oxford, UK: Oxford University Press, 1996, pp. 44-74.

Venkatesh, V., Morris, M. G., Davis, G. B., and Davis, F. D. "User Acceptance of Information Technology: Toward a Unified View," MIS Quarterly (27:3), 2003, pp. 425-479.

Williams, R., and Edge, D. "The Social Shaping of technology," Research Policy (25:6), 1996, pp. $865-899$.

\section{ABOUT THE AUTHORS}

Lee W. McKnight is an associate professor in the School of Information Studies, Syracuse University. He is a research affiliate of the Program on Internet and Telecoms Convergence at MIT, which he founded in 1996. He is also a Research Associate Professor of Computer Science at Tufts University, and President of Marengo Research, a consultancy. Lee's research interests span policy, economic, business, and technical aspects of the emerging global information economy. His research focuses on wireless grids, nomadicity and mobility, networked multimedia, innovation systems, national and international technology policy, the convergence of the Internet and telecommunications industries, and Internet telephony policy. Lee can be reached at 1mcknigh@syr.edu.

Raed M. Sharif is a Ph.D. student in the Information Science and Technology Program, School of Information Studies, Syracuse University. In addition to his background in Economics and Political Science and 5 years of experience in IT management, Raed has an MBA (2002) from Birzeit University, Palestine. His research interests include innovation and development, ICT for development, knowledge and technology transfer, and national and international technology ICT policy and strategy formulation. Raed can be reached at rmalshar@ mailbox.syr.edu.

Lidwien van de Wijngaert is an assistant professor at the Institute for Information and Computing Sciences at Utrecht University in The Netherlands. Currently she is a visiting professor at Syracuse University. Her research focuses on the adoption and use of new technologies such as broadband Internet and wireless grids. Her research always focuses on the perspective of the user. She has published in international journals such as New Media and Society and she is a member and reviewer for the International Communication Association. Recently, she coauthored a textbook on ICT in organizations. Lidwien can be reached at L.vandeWijngaert@cs.uu.nl. 